\title{
Time-varying Doppler Effect formula and its application in Cosmology
}

\section{Time-varying Doppler Effect formula and its application in Cosmology}

\author{
Revised 3/24/2021 18:28:00
}

\author{
Qian Chen \\ International Education Foundation \\ 4667 Highland Ct, Grandville, MI, USA 49418 \\ e-mail: georgechen@iefstudy.org
}

\begin{abstract}
The Doppler effect for electromagnetic waves results in either a redshift or blueshift of light and is of great use in astronomy. For example, it has been used to measure the speed of stars and galaxies approaching or receding from the earth. Currently, all Doppler effect formulas only work for constant velocities. Hence, the application of the Doppler effect includes the implicit assumption of a constant velocity of the motion during the period from the light emission to measurement. Since the light from remote stars detected from Earth may be from millions of years ago, it is difficult to assume that these stars kept moving at constant velocity for the long period, which may become a potential concern. A timevarying Doppler effect formula is mathematically derived from the principle of constant light speed, which is consistent with the classical and the redshift formulas. This formula is also supported by existing experiments and theoretically proved by Maxwell's wave equations. The potential application of this timevarying Doppler effect formula in cosmology is discussed with the examples of cosmological redshift and Hubble's law. The paper shows that the cosmological redshift can be interpreted as a special case of the time-varying Doppler effect. Further discussion between the observed Hubble's redshift relationship and Hubble's law may be needed.
\end{abstract}

Keywords: Doppler effect, Light speed, Redshift, Cosmological redshift, Hubble's law, Asymmetry Theory

\section{INTRODUCTION}

The Doppler effect is the change in frequency of a wave in relation to an observer who is moving relative to the wave source (Doppler 1842 [1]). In 1901, the optical Doppler effect is verified in the laboratory using a system of rotating mirrors [2]. The Doppler effect is of great use in Astronomy with the first application of measuring the velocity of a star moving away from the Earth in 1868 [3]. It was also known as redshift and blueshift in astronomy. A redshift is a decrease in the frequency of light. The opposite change, an increase in frequency, is known as blueshift. Traditionally, the cosmological redshift is believed not Doppler effect, but due to the expansion of space [13].

There are different Doppler effect formulas, for example, the classic formula, the redshift formula used in Astronomy, and the relativistic Doppler effect formula [6] following the Special Relativity [5]. Currently, all the Doppler effect formulas are for constant velocity only. Hence, the application of the Doppler effect includes the implicit assumption of a constant velocity during the period from the light emission to measurement. This is a potential concern for the application in Astronomy, since the light from remote stars detected from the Earth today may be emitted from millions of years ago. It is difficult to assume that the velocity of stars remained the same during such a long period.
The well-established principle of the constancy of the velocity of light (Einstein 1905 [5]), stated: "in empty space, light is always propagated with a definite velocity $\mathrm{V}$ which is independent of the state of motion of the emitting body". This principle can be mathematically represented with the general equation $\boldsymbol{O}\left(t_{o}\right)-\boldsymbol{S}\left(t_{s}\right)=\boldsymbol{c}\left(t_{o}-t_{s}\right)$. Based solely on this equation and without including any other assumptions, a comprehensive set of results named Asymmetry Theory, including a formula of the timevarying Doppler effect, is mathematically derived in [7]. The design of two experiments was proposed in [8] to conclusively confirm the validity of the theory.

First, the asymmetry between the times of light emission and observation is investigated to explain the phenomenon that: when an observer moving away from a clock, the clock appears ticking slower and when the velocity approaches the light speed, he will see the clock coming to a stall. If he compares the observed clock with his clock, he will see a scaling factor. This is due to the delay between the time of light emission as measured by the light source, $t_{s}$, and the observed time as measured by the observer, $t_{o}$, which results in a time scaling factor, $d t_{o} / d t_{s}$. A formula of this scaling factor is mathematically derived in [7].

The Doppler effect is simply a phenomenon of this time scaling factor. A clock ticking slower/faster is equivalent to an "observed" lower/higher frequency. A general formula is mathematically derived in [7] from the time scaling factor 


\section{Time-varying Doppler Effect formula and its application in Cosmology}

$d t_{o} / d t_{s}$, which covers traditional and transverse Doppler effects and applies to time-varying velocities. This formula predicts no frequency shift for circular motion, which is supported by experiments $[9,10]$.

Furthermore, Maxwell's equations [15] provide the theoretical proof of this formula of the Doppler effect. A generalized form of Maxwell's wave equations for moving observers is mathematically derived from the original Maxwell equations in [7], and this Doppler effect formula is mathematically derived from Maxwell's wave equations.

The formula of the cosmological redshift is mathematically derived from the time-varying Doppler effect formula, which shows that the Cosmological redshift can be attributed as a special case of the Doppler effect.

Hubble's law [4] was derived from the observed relationship between the redshift and the distance of the galaxy. This time-varying Doppler effect formula shows that the derivation of Hubble's law was valid only if the recessionary velocity is constant. If the recessionary velocity is time-changing, there may be other theoretically plausible explanations of the observed redshift relationship. Hence, a further discussion may be warranted.

\section{DEFINITION AND PRINCIPLE}

Define $t_{s}$ as the time that the light was emitted; $S\left(t_{s}\right)$ as the origin of the light emitted at $t_{s} ; t_{o}$ as the time an observer $\boldsymbol{O}$ observed the light from $\boldsymbol{S}\left(t_{s}\right) ; \boldsymbol{O}\left(t_{o}\right)$ as the position of $\boldsymbol{O}$ at $t_{o}$. Define $\boldsymbol{v}_{\boldsymbol{o}}(t)$ as the velocity of the observer $\boldsymbol{O}$ relative to $\boldsymbol{S}\left(t_{s}\right)$ and $\boldsymbol{v}_{\boldsymbol{s}}\left(t_{s}\right)$ as the velocity of the light emitter relative to the light origin $S\left(t_{s}\right)$ at $t_{s}$. Fig. 1 shows key angles for the velocity of light, $\theta_{s}, \theta_{o}, \phi$.

The principle of the constancy of light velocity [1] can be represented with the general equation [7]:

$$
\boldsymbol{O}\left(t_{o}\right)-\boldsymbol{S}\left(t_{s}\right)=\boldsymbol{c}\left(t_{o}-t_{s}\right)
$$

The time-varying Doppler effect formula is mathematically derived from (1) without any other assumptions.

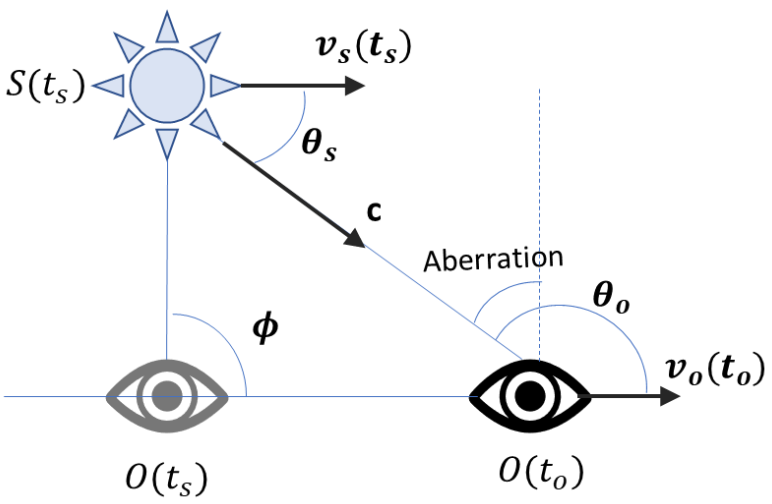

FIG. 1. Key angles of light velocity [7]

\section{VARYING PROPAGATION DELAY}

The motions of the light source and observer created a varying propagation delay between the time of light emission as measured by the light source, $t_{s}$, and the observed time as measured by the observer, $t_{o}$. This varying delay results in a time scaling factor, $d t_{o} / d t_{s}$, which explains the phenomenon that a clock appears ticking slower/faster to an observer moving away from/towards the clock.

Perform an inner product of both sides of (1), we have

$$
\left(\boldsymbol{O}\left(t_{o}\right)-\boldsymbol{S}\left(t_{s}\right)\right) \cdot\left(\boldsymbol{O}\left(t_{o}\right)-\boldsymbol{S}\left(t_{s}\right)\right)=c^{2}\left(t_{o}-t_{s}\right)^{2}
$$

Differentiate both sides as to $t_{s}$

$$
\begin{gathered}
\left(\boldsymbol{O}\left(t_{o}\right)-\boldsymbol{S}\left(t_{s}\right)\right) \cdot\left(\boldsymbol{v}_{o}\left(t_{o}\right) \frac{d t_{o}}{d t_{s}}-\boldsymbol{v}_{s}\left(t_{s}\right)\right)=c^{2}\left(t_{o}-t_{s}\right)\left(\frac{d t_{o}}{d t_{s}}-1\right) \\
\Rightarrow \frac{\left(\boldsymbol{O}\left(t_{o}\right)-\boldsymbol{S}\left(t_{s}\right)\right)}{c\left(t_{o}-t_{s}\right)} \cdot\left(\boldsymbol{v}_{o}\left(t_{o}\right) \frac{d t_{o}}{d t_{s}}-\boldsymbol{v}_{s}\left(t_{s}\right)\right)=c\left(\frac{d t_{o}}{d t_{s}}-1\right)
\end{gathered}
$$

Let $\boldsymbol{i}_{\text {os }}$ denote the unit vector in $\overrightarrow{\mathbf{S}\left(\mathrm{t}_{\mathrm{s}}\right) \mathbf{O}\left(\mathrm{t}_{\mathrm{s}}\right)}$, we have

$$
\boldsymbol{i}_{o s} \cdot \boldsymbol{v}_{o}\left(t_{o}\right) \frac{d t_{o}}{d t_{s}}-\boldsymbol{i}_{o s} \cdot \boldsymbol{v}_{s}\left(t_{s}\right)=c\left(\frac{d t_{o}}{d t_{s}}-1\right)
$$

Finally, the formula for the time scaling factor is [7]

$$
\frac{d t_{o}}{d t_{s}}=\frac{c-v_{s}\left(t_{s}\right) \cos \left(\theta_{s}\right)}{c-v_{o}\left(t_{o}\right) \cos \left(\theta_{o}\right)}
$$

When $v_{\mathrm{o}}(\mathrm{t}), v_{\mathrm{s}}(\mathrm{t})$ are constant $v_{o}, v_{s}$ in the same direction as $\boldsymbol{c}$, i.e. $\theta_{\mathrm{s}}, \theta_{\mathrm{o}}=0,(10)$ reduces to [7]

$$
\frac{d t_{o}}{d t_{s}}=\frac{c-v_{s}}{c-v_{o}}
$$

\section{THE DOPPLER EFFECT}

\section{A. Time-Varying Doppler Effect Formula}

The Doppler effect is shown in [7] as simply the phenomenon of the time scaling factor $d t_{o} / d t_{s}$, and a straightforward mathematical derivation from (2). Because the total wavenumber emitted during the period $d t_{s}$ should be equal to that received during the period $d t_{o}$, we have the following equation:

$$
f_{o}\left(t_{o}\right) * d t_{o}=f_{s}\left(t_{s}\right) * d t_{s}
$$

Hence,

$$
\frac{f_{s}\left(t_{s}\right)}{f_{o}\left(t_{o}\right)}=\frac{d t_{o}}{d t_{s}}
$$

Substitute with the time dilation formula (2), we get the general formula of the time-varying Doppler effect [7]: 


\section{Time-varying Doppler Effect formula and its application in Cosmology}

$$
\frac{f_{s}\left(t_{s}\right)}{f_{o}\left(t_{o}\right)}=\frac{c-v_{s}\left(t_{s}\right) \cos \left(\theta_{s}\right)}{c-v_{o}\left(t_{o}\right) \cos \left(\theta_{o}\right)}
$$

\section{B. Experiments Support}

When the velocities $\boldsymbol{v}_{o}(t), \boldsymbol{v}_{s}(t)$ are constants, $v_{o}, v_{s}$ and in the light propagation direction, formula (6) reduces to the classical Doppler Effect formula [16]:

$$
\frac{f_{s}}{f_{o}}=\frac{c-v_{s}}{c-v_{o}}
$$

When $v_{o} \ll \mathrm{c}$ formula (6) approximates:

$$
\frac{f_{s}}{f_{o}} \approx 1-\frac{v_{s}-v_{o}}{c}
$$

which is the redshift formula used in Astronomy.

Since the Doppler Effect is a direct result of the varying propagation delay of light, the Asymmetry Theory in [7] predicts that there is no frequency shift when the distance between the observer and the light source is constant, such as a circular movement. The relativistic Doppler effect [6] states a frequency shift for circular movement. The experiments of transverse Doppler shift in $[9,10]$ agreed with the prediction in [7].

In summary, the time-varying Doppler Effect formula (6) is consistent with the classical and the redshift formulas. It is also supported by the experiments of the transverse Doppler effect $[9,10]$.

\section{MAXWELL'S EQUATIONS}

The Doppler effect formula (7) can be mathematically derived from the generalized Maxwell wave equations in [7], based on the formula of varying propagation time delay (3), which theoretically validates the formula.

\section{A. Generalized Maxwell Wave Equations}

Maxwell's standard wave equations [15] in a vacuum and charge-free space are:

$$
\begin{aligned}
& \nabla^{2} \mathrm{E}(\mathbf{r}, \mathrm{t})-\frac{1}{c^{2}} \frac{\partial^{2}}{\partial t^{2}} \mathrm{E}(\mathbf{r}, \mathrm{t})=0 \\
& \nabla^{2} \mathrm{~B}(\mathbf{r}, \mathrm{t})-\frac{1}{c^{2}} \frac{\partial^{2}}{\partial t^{2}} \mathrm{~B}(\mathbf{r}, \mathrm{t})=0
\end{aligned}
$$

In a reference frame static to the light origin, $t$ is in the same scale as the clock in the light origin, i.e. $t_{s}$. Equation (9) can be rewritten as [7]:

$$
\nabla^{2} \mathrm{E}\left(\mathbf{r}, \mathrm{t}_{\mathrm{s}}\right)-\frac{1}{c^{2}} \frac{\partial^{2}}{\partial t_{s}{ }^{2}} \mathrm{E}\left(\mathbf{r}, \mathrm{t}_{\mathrm{s}}\right)=0
$$

From the perspective of an observer, only the time it detects the light, i.e. $t_{o}$, can be used to measure the wave propagation. Assume an observer with a velocity $v_{o}$ along the direction of the wave propagation. From (3), we have

$$
\frac{d t_{o}}{d t_{s}}=\frac{c}{c-v_{o}}
$$

Substitute $t_{s}$ with $t_{o}$ in (22), we have [7]

$$
\nabla^{2} \mathrm{E}\left(\mathbf{r}, \mathrm{t}_{\mathrm{o}}\right)-\frac{1}{\left(c-v_{o}\right)^{2}} \frac{\partial^{2}}{\partial t_{o}{ }^{2}} \mathrm{E}\left(\mathbf{r}, \mathrm{t}_{\mathrm{o}}\right)=0
$$

Similarly,

$$
\nabla^{2} \mathrm{~B}\left(\mathbf{r}, \mathrm{t}_{\mathrm{o}}\right)-\frac{1}{\left(c-v_{o}\right)^{2}} \frac{\partial^{2}}{\partial t_{o}{ }^{2}} \mathrm{~B}\left(\mathbf{r}, \mathrm{t}_{\mathrm{o}}\right)=0
$$

(13) and (14) are the generalized Maxwell wave equations as to the moving observers, which are mathematically equivalent to the original Maxwell equations (9) and (10), simply in another form using the time $t_{o}$ from the perspectives of the moving observer. When $v_{o}=0$ they reduce to the original Maxwell equations.

\section{B. Derived Doppler Effect formula}

The general solution to (13) is a linear superposition of waves of the form:

$$
\mathrm{E}\left(\mathbf{r}, t_{o}\right)=\mathrm{g}\left(2 \pi f_{o} t_{o}-\mathbf{k} \bullet \mathbf{r}\right)
$$

where $f_{o}$ is the frequency observed by the moving observer, $\mathbf{k}$ is the wave vector, and $k=|\mathbf{k}|$ is the wavenumber. From (13), $f_{o}, k$ shall satisfy

$$
k^{2}-\frac{1}{\left(c-v_{o}\right)^{2}}\left(2 \pi f_{o}\right)^{2}=0
$$

Hence, the observed frequency $f_{o}$ is

$$
f_{o}=\left(c-v_{o}\right) * k / 2 \pi
$$

Since the emission frequency is $f_{s}=c * k / 2 \pi$, we have:

$$
\frac{f_{o}}{f_{s}}=\frac{c-v_{o}}{c}
$$

which is the same as the Doppler effect formula (7).

\section{THE COSMOLOGICAL RED-SHIFT}

Cosmological red-shift is traditionally believed to be the effect of the inflating Universe [12] and different from the Doppler Effect [13]. Assuming $a(t)$ is the cosmic scale factor, the formula of cosmological red-shift is:

$$
\frac{f_{s}\left(t_{s}\right)}{f_{o}\left(t_{o}\right)}=\frac{a\left(t_{o}\right)}{a\left(t_{s}\right)}
$$

The key problem of integrating the cosmological redshift with the Doppler effect before is that there is a timevarying factor in cosmological redshift, i.e. $a(t)$, while 


\section{Time-varying Doppler Effect formula and its application in Cosmology}

there is not any time factor in current Doppler effect formulas. Now with the time-varying Doppler effect formula (6), it is easy to prove that the cosmological redshift is just one special case of the Doppler effect.

Under the assumption of inflating Universe, the general equation (1) becomes:

$$
\boldsymbol{O}\left(t_{o}\right)-\boldsymbol{S}\left(t_{s}\right)=\int_{t s}^{t o} c / a(t) d t
$$

Following the similar mathematical derivation in III., we have the corresponding Doppler effect formula for the inflating Universe:

$$
\frac{f_{s}\left(t_{s}\right)}{f_{o}\left(t_{o}\right)}=\frac{c / a\left(t_{s}\right)-v_{s}\left(t_{s}\right) \cos \left(\theta_{s}\right)}{c / a\left(t_{o}\right)-v_{o}\left(t_{o}\right) \cos \left(\theta_{o}\right)}
$$

When $v_{s}\left(t_{s}\right), v_{o}\left(t_{o}\right) \ll c$

$$
\frac{f_{s}\left(t_{s}\right)}{f_{o}\left(t_{o}\right)} \approx \frac{a\left(t_{o}\right)}{a\left(t_{s}\right)}
$$

which is the same as (14). Hence, the cosmological redshift is a phenomenon of the Doppler Effect.

\section{HUBBLE'S LAW}

Hubble's law [4] stated that galaxies are moving away from the Earth at speeds proportional to their distance, which is often expressed by the equation

$$
v=H_{0} * D
$$

where $H_{0}$ is the Hubble constant of proportionality; $D$ is the "proper distance" to the galaxy; $v$ is the recessionary velocity of the galaxy from the Earth. Hubble constant's value is recently determined at $74.03 \pm 1.42(\mathrm{~km} / \mathrm{s}) / \mathrm{Mpc}$ [14].

It is important to note that the velocity $v$ in Hubble's law is not directly measured. Hubble discovered a rough proportionality between the measurement of the redshift of an astronomical object and its distance from the Earth, and Hubble's law was derived from this redshift relationship by applying the Doppler effect/redshift formula [11]. Hence, the derivation of Hubble's law is based on the implicit assumption that the recessionary velocity $v$ of the galaxy is constant during the period from the light emission to measurement.

Without this assumption, the timing varying Doppler effect formula (6) is approximated with the following redshift formula

$$
\frac{f_{s}\left(t_{s}\right)}{f_{o}\left(t_{o}\right)} \approx 1-\frac{v\left(t_{s}\right)}{c}
$$

Hence, the Hubble's relationship between the redshift and the distance should be translated to the following general relationship between the velocity and the distance:

$$
v\left(t_{s}\right)=H_{0} * D\left(t_{s}\right)
$$

If we assume a constant velocity $v$, this equation (20) is the same as Hubble's law (18).
However, if we assume a time-varying velocity, then the redshift relationship should be explained not only by the distance of the galaxy but also by the timing of emission. Fig.2 shows an example relationship between the recessionary velocity and both the propagation time and distance. As an example, the redshift shows the recessionary velocity of the galaxy $280.1 \mathrm{Mpc}$ away is $18890 \mathrm{~km} / \mathrm{s}$. But it is important to note this recessionary velocity is the velocity of 913.7 million years ago.

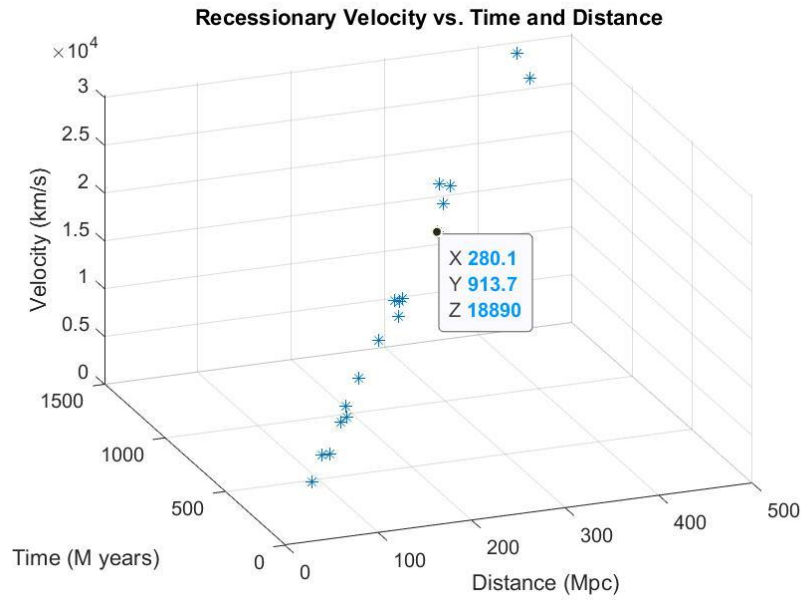

FIG. 2. Recessionary velocity vs. time and distance

To further illustrate, Fig.3 plots the recessionary velocity vs. time and distance separately. If we assume the velocity is invariant of time, Fig.3 is simply a plot of Hubble's law. However, if we consider the extreme assumption that the velocity is unrelated to distance, Fig.3 plots a plausible linear relationship between the velocity and time because the redshift relationship (20) can be rewritten as:

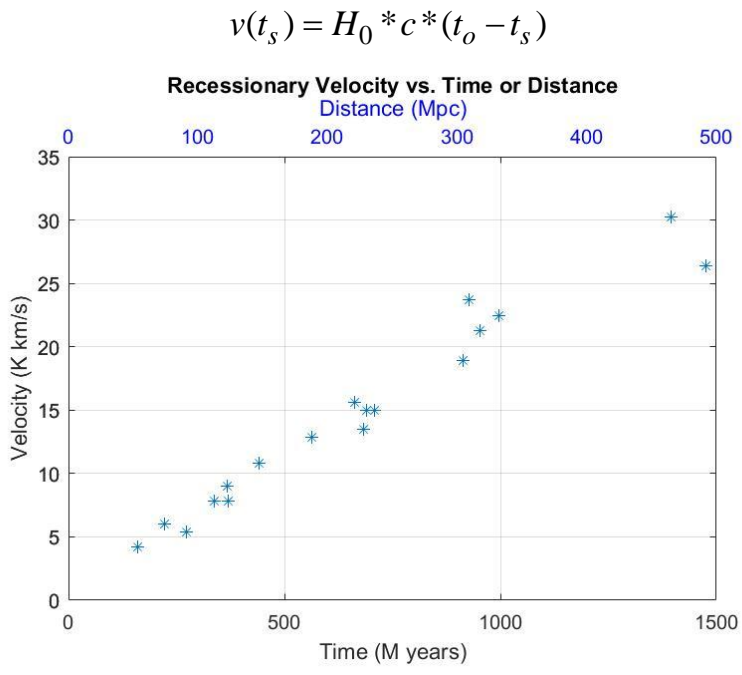

FIG. 3. Recessionary velocity vs. time/distance

To clarify, we don't intend to jump to any conclusion on the relationship of the recessionary velocity. This extreme example is only presented to show the theoretic possibility 


\section{Time-varying Doppler Effect formula and its application in Cosmology}

of other potential explanations of the observed redshift relationship, for example, the redshift may be a result of both the change of recessionary velocity over time and the distance of the galaxy. In other words, Hubble's law can't be simply concluded based solely on the observed redshift relationship unless the constant velocity assumption is supported by other evidence. Hence, a further discussion of Hubble's law may be warranted and additional evidence may need to be considered.

\section{CONCLUSION}

A time-varying Doppler effect formula is mathematically derived, which is consistent with the classical and the redshift formulas. This formula is also supported by existing experiments and theoretically proved by Maxwell's wave equations. The cosmological redshift is shown to be a special case of the time-varying Doppler effect. Hubble's

[1] C. Doppler, Beiträge zur fixsternenkunde, Prague: G. Haase Söhne, 69 (1846).

[2] A. Bélopolsky, On an Apparatus for the Laboratory Demonstration of the Doppler-Fizeau Principle, ApJ. 13, 15 (1901).

[3] W. Huggins, Further Observations on the Spectra of Some of the Stars and Nebulae, Philos. Trans. R. Soc. 158, 529-564 (1868).

[4] E. Hubble, A Relation between Distance and Radial Velocity among Extra-Galactic Nebulae, PNAS., 15 (3), 168-173 (1929).

[5] A. Einstein, On the Electrodynamics of Moving Bodies, Annalen der Physik, 17, 891-921 (1905).

[6] D. Sher, The Relativistic Doppler Effect, J. R. Astron. Soc. Can. 62, 105-111 (1968).

[7] Q. Chen, Asymmetry Theory mathematically derived from the principle of constant light speed, submitted to Found. Phys., doi:10.31219/osf.io/6a74s (2021).

[8] Q. Chen, Design of Experiments for Light Speed Invariance to Moving Observers, submitted to Int. J. Theor. Phys. , doi:10.20944/preprints202102.0547.v1, (2021).

[9] D.C. Champeney et al., Absence of Doppler shift for gamma ray source and detector on same circular orbit., Proc. Phys. Soc. 77, 350 (1961).

[10] H. W. Thim, Absence of the relativistic transverse Doppler shift at microwave frequencies, IEEE Trans. Instr. Measur. 52, 1660-1664 (2003).

[11] E. Harrison, The redshift-distance and velocitydistance laws, ApJ. 403, 28-31 (1992).

[12] A. Eddington, The Expanding Universe: Astronomy's 'Great Debate', 1900-1931, Cambridge University Press (1933).

[13] T. Koupelis et al., In Quest of the Universe, Jones \& Bartlett Publishers, 557 (2007).

[14] A. Ananthaswamy, Best-Yet Measurements Deepen Cosmological Crisis, Scientific American (2019). law may need to be further investigated with additional evidence to conclude the relationship between the recessionary velocity and the distance of the galaxy.

\section{ACKNOWLEDGEMENTS}

This research is sponsored by the International Education Foundation.

\section{DATA STATEMENTS}

The data that supports the findings of this study are available within the article.

[15] J. Maxwell, A Dynamical Theory of the Electromagnetic Field, Philos. Trans. R. Soc. Lond. 155, 459-512 (1865).

[16] N. Giordano, College Physics: Reasoning and Relationships, Cengage Learning, 421-424 (2009). 